\section{Reward System Features Opinion}

Reward system is the overall including all the neurons basis for life functions that you can see summarized into our symbol.

Like all cells it has electric activity and charge, where synchronicity is one of the physical laws explaining some before stupefying unknown obvious characteristics and basis to get to life phoenomena like soulmates or similar natural examples. So on the whole we can say that it has a resultant electric force.

In the detail, every cell and neuron has specifical rest charge, usually $-60 \mathrm{mV}$, balance of ion fluxes into out the cell like $\mathrm{Na}+\mathrm{Cl}-$ $\mathrm{K}+$ that at most determine the cell electric camp. So we can say that, like demonstrated in EEG, brain is an organ with electric activity and camp, with electric energy and waves and magnetic possibilities, seen also from molecular point of view. There are along the reward system, directions of informations and electric vettori and charges. This is positive for life, when they are mostly positively active and life oriented guaranteeing.

The electric function is automatical basis and linked with the chemical molecular activities: being positive toward rest state leading to positive happy molecules brain body metabolism lile dopamine serotonine, typical of the medial sides, bringing positive signals to the automatical parasympathetical body needs, and also growth factors. This of course at the same time impacts on the cell genetic condition, like epigenetical science explains: positive charge and dopamine improving nucleus genes with transcription of genes and molecules life improving. This is also the animal life human so called conditioning fenomenon, reinforcement of the specie saving behaviours and needs or also duties.

We know from all life cultures and sciences that the coupe is
Volume 8 Issue 4 - 2018

\section{Dora Dragoni}

Nutritionist, Acupuncture fellow at AMAB, Italy

Correspondence: Dora Dragoni, Medical Doctor, Nutritionist, Personal Trainer, Acupuncture Fellow at AMAB, Italy, Tel: +39338-508573I; Email dora.dragoni@studio.unibo.it

Received: June 03, 2017 | Published: July 26, 2018

the main exclusive reality to create the couple equilibrium from the reward point of view, being the reward shared in couple not only from jing love life need but also from mental one (since the conscious brain confirms what automatical yang life phase determines: the passage from yuan time to the couple creation for life, then prole creating autonomizing and new spring times for all).

For these reasons reward is personal:

a) Since its obvious from electrical points of view, the more we link positive charges to what we can male positive the best it is for our life.

b) Since epigenetic has to be in couple like billions years natural life has been and will.

In practical meaning: reward are happy molecules positive toward life needs, like our symbol explains. Of course medial reward has to prevale amongst lateral derivations like HPA hormons storage nuclei etc. For this symbol is our answer to the human rights and life bisogni topic for world wellbeing, cfr our bibliografy. 